\title{
High Altitude Plume Simulations for a Solid Propellant Rocket
}

\author{
Jonathan M. Burt ${ }^{1}$ and Iain D. Boyd ${ }^{2}$ \\ Department of Aerospace Engineering \\ University of Michigan, Ann Arbor, MI 48109
}

\begin{abstract}
A simulation scheme is proposed for flowfield and radiation analysis of solid rocket exhaust plumes at high altitude. Several recently developed numerical procedures are used to determine properties of the gas and condensed phase $\mathrm{Al}_{2} \mathrm{O}_{3}$ particles, and spectrally resolved plume radiation calculations are performed using a Monte Carlo ray trace model. Simulations are run for a representative plume flow at $114 \mathbf{~ k m}$, and a comparison is made with experimental measurements of UV radiance. A series of parametric studies involving simulations of this same flow are used to evaluate the influence of physical processes and input parameters related to gas-particle interaction, particle radiation, and the presence of soot.
\end{abstract}

\section{Introduction}

$\mathrm{I}^{\mathrm{n}}$ the flowfield simulation and radiation analysis of solid rocket exhaust plumes at very high altitudes, a number of approximations and simplifying assumptions are typically made due to computational cost, a lack of existing models, or uncertainty over the influence of various physical phenomena. These flows tend to include a large mass fraction of $\mathrm{Al}_{2} \mathrm{O}_{3}$ particles, which can significantly influence bulk flow properties and dominate plume radiative emission through much of the IR, visible, and UV range. Some determination of particle phase characteristics is typically required for useful and accurate simulation results, and may be necessary to assess base heating rates, radiation signatures, surface contamination effects, or other flow properties of interest. As a result, important physical processes and phenomena associated with gas-particle interaction must be recognized and incorporated into simulation procedures. Several potentially important effects have received little attention in the literature, and the significance of coupling between many of these effects still remains an open question. In this paper, we attempt to address the uncertainty in the significance of various effects, and describe a general procedure for the simulation of rarefied plume flows from solid propellant rockets.

At very high altitudes (above roughly $100 \mathrm{~km}$ ) solid rocket motor (SRM) exhaust gases typically experience a high degree of thermal nonequilibrium through much of the plume. As the exhaust gases rapidly expand beyond the nozzle exit, thermal energy is increasingly distributed nonuniformly among the translational and internal degrees of freedom, and the gas velocity distribution may deviate significantly from the equilibrium limit. This is particularly true around the nozzle lip, in the plume backflow region upstream of the nozzle exit plane, and in the plumeatmosphere interaction region where a thick mixing layer may be bounded on either side by highly diffuse shock waves. ${ }^{1}$ Under these conditions the quasi-equilibrium assumptions underlying most continuum CFD methods break down, and alternate simulation techniques valid for transitional flow regimes must be used. Additional procedures are required for inclusion of the $\mathrm{Al}_{2} \mathrm{O}_{3}$ particle phase, which consists of particles in both liquid and solid states with diameters ranging from 0.1 to $10 \mu \mathrm{m}$, and which commonly accounts for $15-30 \%$ of the mass flow through the nozzle. ${ }^{2}$ Momentum and energy exchange between particles and the surrounding gas may significantly influence properties of both phases, and the locally free molecular nature of gas-particle interactions makes a kinetic theory approach more appropriate than traditional continuum methods for two phase flow simulation. Coupling between radiative heat transfer and flowfield characteristics may also be important, as particle phase emission and absorption can significantly affect properties of both the particles and gas.

Over the course of a multi-year project, numerical modeling procedures have been developed for a number of potentially important phenomena associated with high altitude SRM plume flows, and various models have been

\footnotetext{
${ }^{1}$ Post-doctoral research fellow, AIAA member.

${ }^{2}$ Professor, AIAA associate fellow.
} 
individually tested on simple test cases and subscale flows involving a freely expanding plume. ${ }^{3-6}$ For the current effort, a number of these physical models are used together on a larger scale, more complicated flow which better represents the plume flows of interest, and some limited comparison is made between calculated radiation characteristics and available experimental data. Additional simulations are performed for which individual models are disabled or input parameters are varied, in an effort to determine the importance of several physical processes and numerical approximations on flowfield and radiation properties. In the first of these parametric studies, we consider the sensitivity of flowfield and radiation properties to procedures for modeling momentum and energy exchange between condensed phase particles and the surrounding gas. Next, we consider the influence of procedures used in particle radiation modeling. Finally, the potential impact of soot particles on plume radiation properties is examined. Results are presented with the general goal of quantifying the influence and interaction of various phenomena, so that less uncertainty over the importance of these phenomena may exist in future simulation efforts for similar flows.

\section{Numerical Procedures}

To the authors' knowledge, the only experimental measurements for a single-nozzle high altitude SRM plume flow in the open literature are those of Erdman et al. ${ }^{7}$ In a flight experiment termed "Bow Shock Ultra Violet 2" (BSUV-2), onboard sensors were used to measure spectral radiance in the near-UV range (wavelengths of 0.2 to 0.4 $\mu \mathrm{m}$ ) from upper stage SRM exhaust plumes at altitudes between roughly 100 and $120 \mathrm{~km}$. The plume from the third stage Star-27 SRM at an altitude of $114 \mathrm{~km}$ is used as a test case here, and while no experimental data is available on flowfield properties, we can to a limited extent infer overall simulation accuracy from a comparison of radiation characteristics between numerical and experimental results. Following previous simulations of Candler et al. ${ }^{8}$ for the same flow, we perform a series of axisymmetric simulations on a rectangular grid which extends from $10 \mathrm{~m}$ upstream to $75 \mathrm{~m}$ downstream of the nozzle exit plane, and $30 \mathrm{~m}$ radially outward from the nozzle centerline. The nozzle exit diameter is $0.78 \mathrm{~m}$, and the rocket surface geometry is approximated as a blunted cone-cylinder shape of length $3.1 \mathrm{~m}$. The grid geometry is shown along with relevant boundary conditions in Fig. (1).

To account for the high degree of gas thermal nonequilibrium expected through much of the grid domain, the direct simulation Monte Carlo (DSMC) method ${ }^{9}$ is used to calculate gas properties. The variable hard sphere (VHS) collision model is employed to approximate the variation in transport properties with temperature, and the LarsenBorgnakke model ${ }^{9}$ is used together with a total collision energy model for the probability of inelastic collisions to determine energy exchange between translational and rotational modes.

As the gas density in much of the plume core region downstream of the nozzle is far too high to meet standard DSMC cell size requirements on all but the largest parallel machines, an alternate approach for gas phase simulation must be used in this region. The traditional collision-limiter approach provides a simple solution, but is considerably less efficient and arguably less accurate than alternate methods which involve resampling some fraction of representative gas molecule velocities during each time step from predetermined distribution functions. Both types of approaches can produce large artificial diffusion errors, and tend to enforce a near-equilibrium gas velocity distribution that may not be physically justified when cell dimensions are much greater than a mean free path. ${ }^{10}$ However, these approaches are generally far simpler to implement than alternate methods, such as hybrid CFD/DSMC codes, for simulating flowfields that include both continuum regions and nonequilibrium regions in which a DSMC calculation is desired. This is particularly true for simulations involving a large number of additional physical models, or those for which strong two-way coupling may exist between continuum and nonequilibrium regions.

For this study, gas properties in the high density, near-equilibrium core flow region of the plume are calculated using a recently developed particle method based on the ellipsoidal statistical Bhatnagar-Gross-Krook (ES-BGK) equation. ${ }^{11}$ Consideration is made for effects of moderate translational and rotational nonequilibrium, and an exact momentum and energy conservation scheme is used to minimize random walk errors. The location of the boundary between DSMC and ES-BGK domains is determined using a standard cutoff value (0.05) of the maximum gradientlength local Knudsen number. ${ }^{12}$ This is a nondimensional continuum breakdown parameter defined as the maximum ratio of the local mean free path to characteristic length scales based on gradients in translational temperature, bulk velocity or density, and has been used in previous studies to estimate the validity of near-equilibrium simulation methods under a variety of conditions.

Condensed phase particles are included in the simulation here through a standard Lagrangian approach, where representative particles are tracked through the grid and sorted into cells using procedures similar to those in DSMC. The momentum and energy transfer to a particle from the surrounding gas is computed using a method of Gallis et 
al., ${ }^{14}$ based on assumptions of locally free molecular flow and an approximation that all interphase collisions involve either specular reflection or diffuse reflection with full thermal accommodation to the particle temperature. The reciprocal momentum and energy transfer from a particle to the gas is determined through a probabilistic two-way coupling approach. ${ }^{3}$ Modifications to both methods are made to allow for nonspherical particles and to account for effects of particle rotation. ${ }^{4}$ A model for nonequilibrium crystallization of liquid $\mathrm{Al}_{2} \mathrm{O}_{3}$ droplets is implemented as well. ${ }^{5}$

Radiative heat transfer to and from the particles is calculated using a Monte Carlo ray trace (MCRT) radiation model, which involves tracking a large number of representative energy bundles through the computational domain. Energy bundles here represent a large collection of photons over some finite wavelength range, and are created periodically at randomly selected source particles throughout the grid. Additional energy bundles are created along inflow boundaries at the nozzle exit, to account for effects of continuum "searchlight" emission from within the nozzle. This model is strongly coupled to the flowfield simulation, and allows for spectrally resolved radiation calculations in an emitting, absorbing and scattering medium of arbitrary optical thickness. ${ }^{6}$

Following simulations of Anfimov et al., ${ }^{13}$ the $\mathrm{Al}_{2} \mathrm{O}_{3}$ particle phase is assumed to account for $30 \%$ of the mass flow at the nozzle exit, and is made up of seven discrete particle sizes ranging from 0.3 to $6 \mu \mathrm{m}$ in diameter. Properties for each particle size are independently specified along inflow boundaries at the nozzle exit plane. These inflow properties, as well as those of the gas, are taken from a nozzle flow simulation by the same authors. Due to a lack of available flowfield information at the nozzle exit, most particle properties given by Anfimov et al. ${ }^{13}$ are assumed uniform over the exit plane. The one exception is in the initial particle directions; for both particles and gas, bulk velocities along the nozzle exit plane are set to scale linearly with distance from the axis. Particle trajectories and gas streamlines at the nozzle exit will have no radial component along the axis, and will reach a maximum offaxis angle of $17.2^{\circ}$ (equal to the nozzle divergence angle) at the nozzle lip.

A rough estimate of the initial solid mass fraction for each particle size is found by taking the difference between the nucleation temperature $\left(\mathrm{T}_{\mathrm{f}}=1930 \mathrm{~K}\right)$ for homogeneous crystallization of $\mathrm{Al}_{2} \mathrm{O}_{3}$ and the assigned particle temperature, then multiplying this by the ratio of the specific heat to the latent heat of fusion for liquid $\mathrm{Al}_{2} \mathrm{O}_{3}$. Based on this procedure and the initial particle temperatures given by Anfimov et al., at the nozzle exit the largest particles are in a completely liquid state and the smallest particles have a liquid mass fraction of 58\%. Particle properties at the nozzle exit are given in Table (1).

The exhaust gas species are limited here to the primary species $\mathrm{H}_{2}, \mathrm{~N}_{2}$ and $\mathrm{CO}$, with initial mole fractions of $0.38,0.31$ and 0.31 respectively. At atmospheric inflow boundaries shown in Fig. (1), the gas has mole fractions of 0.78 for $\mathrm{N}_{2}$ and 0.22 for $\mathrm{O}_{2}$, with a temperature of $288 \mathrm{~K}$, a density of $5.79 \times 10^{-8} \mathrm{~kg} / \mathrm{m}^{3}$, and a freestream Mach number of 13.5. A thermal accommodation coefficient of 0.9 is used for collisions between gas molecules and $\mathrm{Al}_{2} \mathrm{O}_{3}$ particles, while the outer surfaces of the rocket are modeled as diffusely reflecting walls at $300 \mathrm{~K}$ with full thermal accommodation.

Calculations are run for about 60 hours on eight $1.4 \mathrm{GHz}$ AMD Athlon processors. Domain decomposition is used to roughly evenly divide the computational load among all processors, and cell size, time step and numerical weights are carefully varied throughout the grid to balance overall simulation accuracy with computational efficiency. The unstructured grid is divided into about 87,000 triangular cells, through which around 7.5 million DSMC gas particles and 160,000 representative $\mathrm{Al}_{2} \mathrm{O}_{3}$ particles are tracked at steady state.

\section{Simulation Results}

Figure (2) presents a close-up view of gas bulk velocity contours and streamlines in a $10 \mathrm{~m}$ long region surrounding the rocket. The figure shows a highly diffuse bow shock, with a large boundary layer along the front portion of the rocket surface. Further downstream, a thick mixing layer forms along the region of plume-atmosphere interaction, and a small plume backflow region is found just upstream of the nozzle exit and within a few meters of the rocket. Within the plume, streamlines and velocity contours show the characteristics of a near-free expansion flow - including a complete absence of shocks - as expected for a highly underexpanded plume at very high altitude. Note the well resolved boundary between the plume and atmospheric flow regions.

Contours of average particle temperature are shown in Fig. (3) for 0.4 and $4 \mu \mathrm{m}$ diameter $\mathrm{Al}_{2} \mathrm{O}_{3}$ particles. Note first the difference in the regions occupied by particles of the two different sizes. Smaller particles experience a greater radial acceleration in the plume nearfield region just beyond the nozzle exit, so the maximum divergence angle is greater for the smaller particle size. Also note that the temperature of the smaller $0.4 \mu \mathrm{m}$ particles is uniformly about $1200 \mathrm{~K}$ lower than that of the $4 \mu \mathrm{m}$ particles. This can be explained by dependence on the particle diameter $\mathrm{D}_{\mathrm{p}}$ for particle heat capacity, which scales as $\mathrm{D}_{\mathrm{p}}{ }^{3}$, and the convective heat transfer rate, which is 
proportional to $\mathrm{D}_{\mathrm{p}}{ }^{2}$. It follows from these scaling relations that the rate of change in particle temperature tends to vary as $1 / \mathrm{D}_{\mathrm{p}}$, so that smaller particles will be more rapidly cooled by the surrounding gas in the plume nearfield region where convective heat transfer is significant.

For both particle sizes in Fig. (3), the average temperature is generally found to increase with distance from the central axis. This is due to the fact that most trends in particle temperature are dominated by the influence of convective heat transfer. As the convective heat transfer rate is proportional to the local gas density, we expect that particles which pass through the lower-density portions of the plume further from the axis will experience less heat loss to the cooler surrounding gas. Particles further from the axis will therefore retain higher temperatures, as is shown in the figure. The increase in particle temperatures at points very close to the axis is also related to the radial variation in gas density within the plume nearfield region. Random walk errors in cells near the axis produce an artificially low gas density, which in turn reduces convective heat transfer rates for particles which pass through these cells. (This random walk is a consequence of the near-axis reduction in numerical weights required to populate cells along the axis with a sufficient number of representative gas molecules.)

A close observation of $4 \mu \mathrm{m}$ particle temperature contours in Fig. (3) reveals a rapid temperature jump about 0.4 $\mathrm{m}$ downstream of the nozzle exit. This jump is associated with the initiation of the phase change process, and results from the release of the latent heat of fusion for liquid $\mathrm{Al}_{2} \mathrm{O}_{3}$. As given in Table (1), $4 \mu \mathrm{m}$ particles are assumed to be in a completely liquid state at inflow boundaries along the nozzle exit, and are rapidly cooled by the surrounding gas until they reach the nucleation temperature for homogeneous crystallization. Once this temperature is reached, a radially symmetric crystallization front progresses from the surface of a particle toward its center. The heat release associated with the phase change process causes a sudden increase in particle temperature, and this temperature increase in turn slows the progress of the crystallization front. For some time a quasi-equilibrium state may be reached, where the heat release during crystallization is balanced by convective and radiative heat loss, and the particle maintains a nearly uniform temperature. Further downstream, as the liquid mass fraction approaches zero and the rate of heat release is reduced, the particle temperature again begins to decrease as observed in Fig. (3).

To demonstrate the correlation between phase change and the variation in particle temperatures, a contour plot of liquid mass fraction for larger particle sizes is included here as Fig. (4). The top half of the figure shows cellaveraged liquid mass fractions for $4 \mu \mathrm{m}$ particles, while the lower half gives contours for particles of $6 \mu \mathrm{m}$ diameter. A comparison of characteristics for $4 \mu \mathrm{m}$ particles between Figs. (3) and (4) shows that, as described above, the particle temperature jump about $0.4 \mathrm{~m}$ downstream of the nozzle exit is accompanied by the initiation of phase change and a rapid reduction in the liquid mass fraction. Particle temperatures level off further downstream, as the gradient in the liquid mass fraction decreases, and the particles ultimately approach a completely solid state.

In comparing liquid mass fraction contours for the two different particle sizes shown in Fig. (4), we find that phase change begins significantly further downstream for the larger $6 \mu \mathrm{m}$ particles. As explained above, the rate of temperature decrease due to convective heat transfer tends to vary as roughly the inverse of the particle diameter, so that larger particles will experience a longer residence time in a completely liquid state before reaching the nucleation temperature at which crystallization may begin.

For both 4 and $6 \mu \mathrm{m}$ particles, the onset of crystallization is shown in Fig. (4) to occur progressively downstream for particles which move along trajectories further from the axis. As with the radial variation in particle temperatures, this trend is a result of the decrease in gas density with distance from the axis. Particles which pass through regions of lower gas density will cool more slowly through convective heat transfer, so these particles tend to reach the nucleation temperature at points further downstream.

In Fig. (5), contours are shown for the direction-averaged spectral radiative energy flux $E_{\lambda}$ at wavelengths of 2.2 $\mu \mathrm{m}$ and $0.24 \mu \mathrm{m}$. This flux is defined as the energy transfer rate per unit wavelength from photons within a narrow wavelength range which impinge on a spherical surface, divided by the cross sectional area of the sphere. While $E_{\lambda}$ values have little physical significance outside of the MCRT radiation model ${ }^{6}$ used in the simulation, contours of $E_{\lambda}$ provide a convenient means of quantifying the variation in spectrally resolved radiation intensity throughout the simulated flowfield. The upper half of Fig. (5) can therefore be viewed as a measure of IR radiation intensity through the grid, while the lower half shows the intensity at a representative wavelength in the UV range. In comparing $E_{\lambda}$ contours for the two different wavelengths, we find that the IR radiation intensity is consistently about four orders of magnitude greater than the UV intensity, as follows from the fact that thermal radiative emission at the particle temperatures shown in Fig. (3) is typically concentrated in the IR range. ${ }^{2}$

A significant difference is also found in the shape of contour lines between the two wavelengths shown in Fig. (5). This difference may be attributed to the variation with wavelength in the temperature dependence for thermal emission. At smaller wavelengths the spectral emissive power for a blackbody tends to depend far more strongly on 
the temperature of the body. ${ }^{15}$ This characteristic is preserved in the procedures used to determine emissive power output for source particles in the radiation model. ${ }^{6}$ As observed in Fig. (3), particles further from the axis tend to have higher temperatures, so experience a greater rate of radiative heat loss. This temperature difference has a stronger correlation to emissive power in the UV range than in the IR range, so a more pronounced ridge is shown in the UV energy flux contours near the location of higher temperature particles.

As shown in Fig. (5), the energy flux for both wavelengths is greatest at the nozzle exit, due primarily to the corresponding maximum in particle mass density and a reduction in the intensity of nozzle searchlight emission ${ }^{6}$ with distance from the nozzle. Particles are modeled here as volumetric emitters, ${ }^{2,6}$ so the magnitude of radiative fluxes should scale roughly with the local particle mass density. As this density decreases with downstream distance due to the divergence of particle trajectories through the plume, a continuous reduction in radiative energy flux is found in the axial direction.

A reduction in energy flux is also observed in the radial direction, particularly outside the region where particles are found. This follows from the fact that, in an axisymmetric simulation, the ratio of cell volume to the projected area on the grid will scale with distance from the axis. The average number of energy bundles which pass through a cell is proportional to the projected area of that cell in the grid plane, and the contribution of each bundle to the energy flux is proportional to the inverse of the cell volume. We therefore expect the net energy flux through cells outside the particle domain to scale approximately with the inverse of the distance from the axis, as is shown for both IR and UV wavelengths in Fig. (5).

\section{Comparison with Experiment}

While there are no experimental measurements of flowfield properties which could be used to evaluate the overall accuracy of the simulation, some comparison can be made with experimental data and previous numerical results for radiation properties in the plume. In the BSUV-2 experiment, ${ }^{7}$ an onboard radiometer was used to measure UV spectral radiance along a downstream line inclined $4^{\circ}$ to the nozzle centerline. The MCRT radiation model employed here is used to generate corresponding radiance values at several discrete wavelengths, and a comparison between numerical and measured values is shown in Fig. (6). Data points in the figure labeled "base simulation" are taken as the averages among eight otherwise identical simulations for which the random number generator is initialized at different points. The error bars give 95\% confidence intervals based on a wavelengthaveraged variance from the eight simulations, and show that the stochastic nature of the radiation model produces significant scatter in these radiance values.

Published numerical results of Candler et al. ${ }^{8}$ and Anfimov et al. ${ }^{13}$ are also included in Fig. (6) for comparison. Both of these previous simulations employ continuum CFD methods which for this flow may be less appropriate than the kinetic approach used here, particularly in the highly rarefied regions upstream of the nozzle exit. Still, somewhat better agreement is found with experiment for these results than for those from the present simulations, particularly at wavelengths below around $0.25 \mu \mathrm{m}$. Note however that in the simulations of Candler et al., particle phase enthalpies at the nozzle exit were selected for best agreement between calculated and experimental radiance values at the $0.23 \mu \mathrm{m}$ wavelength. This procedure effectively rescales the UV radiance profile to match experimental results, and makes any quantitative comparison with experiment of questionable value.

In the simulations performed for this study, particle spectral emissivity values are assumed to scale with the ratio of the particle absorption index to the wavelength, following a correlation of Reed and Calia. ${ }^{2}$ Absorption index values are in turn calculated as a function of temperature and wavelength. In the IR range where most radiative energy transfer is concentrated, absorption index values are interpolated from experimental measurements of Konopka et al., ${ }^{16}$ while those in the UV range are found using a series of correlations employed in the plume calculations of Anfimov et al. ${ }^{13}$ These correlations exhibit a strong temperature and wavelength dependence that has not been verified by other authors, and do not account for extrinsic properties (such as impurity concentrations and lattice defects) that have been found to dominate radiation characteristics of solid phase particles. ${ }^{2}$ The resulting uncertainty in radiance values leads to some doubt regarding the validity of using these values to assess overall simulation accuracy.

To determine the effect of the UV absorption index correlations on radiance values, two additional simulations are performed. In the first simulation, the absorption index is given a constant value of 0.01 through the UV range. This value is chosen to roughly characterize an average of values taken from the correlations of Anfimov et al. ${ }^{13}$ over a range of temperatures and wavelengths. In the second simulation, the UV absorption index is set so that the spectral emissivity $\varepsilon_{\lambda}$ in the UV range is approximately constant. It follows from the correlation of Reed and Calia ${ }^{2}$ 
that, for a constant emissivity, the absorption index $\mathrm{k}$ in this range must be proportional to the wavelength $\lambda$. For a given particle radius $R_{p}$, we can relate $k$ to $\lambda$ by

$$
\mathrm{k}=\frac{\varepsilon_{\lambda} \cdot \lambda}{4 \mathrm{R}_{\mathrm{p}}}
$$

Particles are modeled through Eq. (1) as volumetric emitters, ${ }^{2}$ so that in an isothermal enclosure containing particles with a range of sizes, the emissive heat flux per unit volume will scale with the mean-cubed particle radius. As this flux should also scale with the average over all particle sizes of the quantity $\varepsilon_{\lambda} R_{p}{ }^{2}$, an effective emissivity which is independent of the particle radius must be proportional to the ratio of the mean-cubed radius to the mean-squared radius. This ratio is equal to one-half the Sauter mean diameter, which may be substituted for $R_{p}$ in Eq. (1) for use with an effective emissivity which is independent of particle size.

Thus, to allow for values of the UV absorption index which give a uniform effective emissivity, we assume a characteristic Sauter mean diameter of $3.2 \mu \mathrm{m}$ (as is found through much of the plume region occupied by particles) and use an emissivity of 0.2. From Eq. (1), this corresponds to the relation $\mathrm{k}=0.031 \lambda$. The resulting $\mathrm{k}$ values for this "constant emissivity" case are generally of the same order as those found through the correlations of Anfimov et al., ${ }^{13}$ and differ little from values used in the simulation involving a constant absorption index.

Radiance values from both additional simulations are shown in Fig. (6), and exhibit significantly better agreement with experiment than do base simulation results. In particular, the constant emissivity simulation gives excellent agreement with the experimental data at smaller wavelengths where the correlations of Anfimov et al. produce the greatest disagreement. While the choice of constant emissivity or absorption index values is to some extent arbitrary, the resulting consistency with experiment gives us reasonable confidence in the overall accuracy of the simulations.

\section{Sensitivity to Gas-Particle Interaction Models}

The simulations presented here involve a large number of modeling procedures, covering a range of physical phenomena, for which the sensitivity of simulation results to input parameters may not be known. The importance of coupling between the various models is also generally uncertain, and assumptions underlying several of these models may not always be valid under relevant flowfield conditions. As the choice of input parameters and modeling approximations is of great importance in assessing the overall accuracy of the simulation approach, some evaluation is desired for the relative contributions and interactions among modeling procedures used in the simulations. For this evaluation, results from the base simulation described above are compared with those from a series of simulations for which individual models are turned off or input parameters are varied. The first category of physical models and numerical approximations to be assessed are those involved in momentum and energy exchange between the particles and gas.

First, the influence of momentum and energy coupling from particles to the surrounding gas is considered by deactivating the two-way interphase coupling model, ${ }^{3}$ then performing a simulation for which gas properties are not affected by the presence of the particle phase. The effects of particle rotation are considered next, through the use of models for the exchange of angular momentum, linear momentum and energy between a rotating sphere and a locally free molecular nonequilibrium gas. ${ }^{4}$ Particle angular velocities at the nozzle exit are given random directions along a plane normal to the nozzle centerline, and are assigned magnitudes based on an estimate of the maximum angular velocity which avoids centrigufal breakup in liquid $\mathrm{Al}_{2} \mathrm{O}_{3}$ droplets. (Details of the procedure are provided in Ref. 4.) These droplets are assumed to rotate as a result of off-center coalescing collisions within the combustion chamber and nozzle.

Another property considered here is the influence of particle shape: While $\mathrm{Al}_{2} \mathrm{O}_{3}$ particles extracted from SRM exhaust flows have been found to be roughly spherical, some authors have discussed an expected deviation of particle shapes from a perfect sphere due to surface forces on a solidifying droplet. ${ }^{2}$ To quantitatively evaluate effects of particle shape, a simulation is performed for which particles are assumed to have the same surface-area-tovolume ratio as a cube. Following a detailed analysis of momentum and energy transfer rates between a rotating nonspherical particle and a locally free-molecular gas, ${ }^{3}$ the effective particle diameter and material density are modified in order to approximate the influence of a cube-shaped surface. While this represents a deviation from spherical shape well out of the expected range, a comparison with base simulation results can be used to determine outer bounds for the influence of particle shape on various flowfield properties.

A final characteristic of gas-particle interaction considered here is the effect of the thermal accommodation coefficient (TAC) at the particle surface. For all simulations described above, the particle TAC is set to 0.9, so that $90 \%$ of interphase collisions involve diffuse reflection with full accommodation to the particle temperature, and the 
remaining $10 \%$ involve specular reflection. While this TAC value is within the general range observed experimentally, the phenomenological nature of the collision model and a potentially strong dependence on surface and gas properties lead to some uncertainty over what value would be most appropriate. To assess the influence of the TAC value on flowfield properties, an additional simulation is performed for which the TAC is set to 0.45 , so that interphase collisions are half as likely to involve diffuse reflection as in the base simulation.

Results from these simulations are compared by extracting flowfield properties along a straight line which roughly follows the gas streamline originating midway between the nozzle lip and the intersection of the central axis with the nozzle exit plane. In the coordinate system of Fig. (1), the extraction line extends from the point $x=0, y=$ 0.2 to $\mathrm{x}=75, \mathrm{y}=25$, where all values are in meters. This line is chosen to give a general sense of the downstream variation in gas and particle properties through the plume, while avoiding regions near the central axis which are prone to relatively large statistical scatter. Results are shown in Figs. (7) through (11).

In Fig. (7), the gas translational temperature for each of the simulations described above is plotted as a function of distance from the nozzle exit along the extraction line. The temperature variation for the base simulation shows generally expected trends for a plume flow expanding into a near-vacuum: The largest temperature gradient occurs in the nearfield region just downstream of the nozzle exit, following a rapid conversion of thermal energy into bulk kinetic energy within this region. The rate of change slowly decreases with downstream distance as collisional processes become progressively less significant, and the translational temperature asymptotically approaches a farfield value near $0 \mathrm{~K}$. Note that effects of rotational freezing allow for a potentially much higher asymptotic rotational temperature.

In comparing results with those from the base simulation, the one-way coupling case is found to give significantly lower gas translational temperatures, particularly within the nearfield plume region close to the nozzle exit. When the heat transfer from $\mathrm{Al}_{2} \mathrm{O}_{3}$ particles to the cooler surrounding gas is neglected, the gas temperature may be reduced within this region by well over $100 \mathrm{~K}$. In contrast, the effect of particle rotation is found here to be negligible, as the gas temperature is shown to be nearly identical between the base simulation and the simulation for which particles leave the nozzle with maximum possible angular velocities.

Particle shape is shown in Fig. (7) to have a potentially measurable, although small, influence on gas temperatures through much of the plume. When particles of fixed mass are given greater surface area, the interphase collision frequency will increase, and higher gas temperatures will result from a corresponding rise in convective heat transfer rates between the particles and gas. The simulation for which the particle TAC is set to 0.45 also gives translational temperature values noticeably different from those of the base simulation. When the probability that an interphase collision involves diffuse reflection is halved, the heat transfer rate between the particles and gas will be reduced by roughly $50 \%$, and particles will be considerably less effective in heating the surrounding gas. As a result, the gas translational temperature is found to decrease by up to approximately $50 \mathrm{~K}$ when the TAC is varied from 0.9 to 0.45 .

Average temperatures along the extraction line for $0.4 \mu \mathrm{m}$ diameter particles are shown in Fig. (8). The temperature plot for the base simulation shows a rapid drop in temperature around the nozzle exit, and a temperature gradient which decreases continuously with downstream distance. An inspection of heat transfer rates shows that the downstream reduction in particle temperatures occurs primarily as a result of convective heat transfer in the nearfield plume region within about $5 \mathrm{~m}$ of the nozzle exit, while radiative heat transfer dominates further downstream. In comparing results between the base simulation and the one-way coupling simulation, for which effects on the gas of interphase momentum and energy exchange are neglected, we find trends similar to those described above for the gas temperature. As the gas temperature is uniformly lower when one-way coupling is assumed and particles are unable to heat the surrounding gas, the local temperature difference between particles and gas will increase. A larger temperature difference corresponds to a greater convective heat transfer rate, so that particles will cool more rapidly and maintain lower temperatures, as is shown in the figure.

As with gas temperatures, the temperature of $0.4 \mu \mathrm{m}$ particles is found to be almost completely unaffected by particle rotation. Particle temperatures are however shown to decrease slightly when given a nonspherical shape. Nonspherical particles will have a larger average collision cross section than spherical particles of equal mass, so will cool more rapidly as a result of convective heat transfer. Note that, as mentioned in the discussion of Fig. (5), particles are modeled as volumetric emitters, so particle shape will have no effect on radiative heat transfer rates other than that associated with the difference in temperatures shown in Fig. (8). From the temperature plot for the simulation where TAC $=0.45$, we find that the TAC value significantly influences $0.4 \mu \mathrm{m}$ particle temperatures throughout the plume. As a result of the roughly $50 \%$ reduction in convective heat transfer rates for this simulation, the temperature of $0.4 \mu \mathrm{m}$ particles is found to be uniformly about $100 \mathrm{~K}$ higher than in the base simulation. 
Figure (9) shows the variation along the extraction line in average temperatures for $4 \mu \mathrm{m}$ diameter particles. The nearfield temperature jump observed for all simulations here is a consequence of the nonequililibrium crystallization process, as explained above in the discussion of Figs. (3) and (4). A comparison between the base simulation results in Fig. (9) and those from the other simulations shows some surprising characteristics: When the TAC is reduced, convective heat transfer rates decrease and the location at which phase change begins is moved further downstream. While this results in higher particle temperatures in the farfield plume region, the delay in phase change initiation leads to significantly lower particle temperatures in a small region upstream of the temperature spike. This region, where a lower TAC corresponds to reduced particle temperatures, is shown in the figure at locations between 0.5 and $1.1 \mathrm{~m}$ from the nozzle exit. Likewise, the increase in convective heat transfer rates associated with both nonspherical particles and an assumption of one-way coupling tends to force the phase change initiation point upstream. Note however that the one-way coupling simulation gives farfield particle temperatures nearly identical to those from the base simulation, while particle shape effects are found to be potentially significant throughout the plume.

As in Figs. (7) and (8), particle rotation is shown in Fig. (9) to have no noticeable effect on $4 \mu \mathrm{m}$ particle temperatures. This is in fact true of all flowfield characteristics considered, so we can conclude that particle rotation can generally be neglected in simulations of similar SRM plume flows, with no impact on simulation results. This conclusion is however subject to assumptions regarding the mechanisms by which particles develop angular momentum. If collisions involving partially or fully solidified particles are considered, the appropriate value of the maximum angular velocity magnitude may be far higher. Higher angular velocities may also result from consideration of the interaction between solid particles and either nozzle walls or large-scale turbulent structures within the nozzle.

Figure (10) shows the variation in the net direction-averaged radiative energy flux along the extraction line. This flux is defined as the integral of the spectral radiative energy flux, displayed in the contour plots of Fig. (5), over the entire wavelength range $(0.2$ to $4.5 \mu \mathrm{m})$ considered in the radiation model. As described in the discussion of Fig. (5), the radiative energy flux is found to decrease continuously with downstream distance through the plume, primarily as a result of the divergence of particle trajectories. The greatest variation from base simulation results is observed for the simulation with TAC $=0.45$, for which the energy flux through much of the farfield plume region is significantly higher. Much of this increase can be attributed to the difference in temperatures for $4 \mu \mathrm{m}$ particles between the two simulations. These particles are shown in Fig. (9) to experience the greatest temperature difference about $25 \mathrm{~m}$ downstream of the nozzle exit. This location corresponds nearly exactly to the point in Fig. (10) where the maximum difference in energy flux is observed. As $4 \mu \mathrm{m}$ particles make up roughly $60 \%$ of the total $\mathrm{Al}_{2} \mathrm{O}_{3}$ mass in the plume, and as the net emissive heat transfer rate increases with particle temperature, this correlation between trends shown in Figs. (9) and (10) is both logical and expected.

A similar correlation between the two figures is observed in comparing results for the base simulation and the simulation involving nonspherical particles. Spherical particles of $4 \mu \mathrm{m}$ diameter are found in Fig. (9) to maintain significantly higher temperatures through much of the plume than nonspherical particles of equal mass. This temperature difference corresponds to a noticeable reduction in the radiative energy flux when nonspherical particles are used. In contrast, simulations involving one-way interphase coupling and rotating particles are both shown in Fig. (10) to give energy flux values which are, within the scale of the figure, identical to those taken from base simulation results.

Note in Fig. (9) that the variation in TAC has a significant effect on $4 \mu \mathrm{m}$ particle temperatures in the nearfield plume region within a few meters of the nozzle exit, although no similar effect is found on energy flux values in this region. Nearfield differences in the temperature of $4 \mu \mathrm{m}$ particles when two-way coupling is disabled, or when nonspherical particles are employed, are also not reflected in Fig. (10). The lack of a correlation here between particle temperatures and energy flux is likely due to some combination of factors: First, smaller particles cool more quickly than larger $4 \mu \mathrm{m}$ particles, so the relative influence of smaller particles on radiative energy flux will be greater at locations further upstream. Second, the net radiative energy flux at any point in the flowfield is a function of particle temperatures throughout the plume, so that contributions to the energy flux associated with large temperature variations in the nearfield plume region will to some extent be "smoothed out" over the length of this region. Finally, nozzle searchlight emission ${ }^{6}$ is expected to have a significant influence on the energy flux near the nozzle exit, so that $\mathrm{Al}_{2} \mathrm{O}_{3}$ particle emission will have a comparatively smaller effect on flux values here than at locations further downstream.

In Fig. (11) UV spectral radiance values at the onboard sensor are shown for the base simulation and for all simulations for which gas-particle interaction models are varied. The measured UV radiance profile from the 
BSUV-2 flight experiment is also included here for reference. Ideally a comparison of radiance values from these simulations would give us some insight as to which numerical approximations and assumptions contribute most significantly to the discrepancy between simulation and experimental results. However, no clear trends are observed among the calculated radiance values, and through most of the wavelength range shown here, the variation between results from the different simulations is within the level of statistical uncertainty given by the error bars. We can at least conclude from this comparison that none of the modeling assumptions evaluated in this section are alone responsible for the discrepancy between experimental and calculated UV radiance profiles.

\section{Influence of Radiation Modeling Parameters}

In addition to numerical approximations and assumptions used for gas-particle interaction, overall simulation accuracy is subject to a number of approximations in the MCRT radiation model. To assess the influence of radiation modeling procedures on flowfield properties and on the plume radiation signature, further simulations are performed for which elements of flowfield-radiation coupling are disabled or input parameters for the radiation model are modified.

First, the influence of radiative absorption is neglected by modifying the code so that MCRT energy bundles have no influence on the temperature of particles assigned to cells through which the energy bundles pass. This in effect decouples radiative absorption calculations from the flowfield simulation. Next, a simulation is run using the traditional post-processing approach to plume radiation analysis, where all radiative terms are neglected in the particle energy balance.

Results from additional simulations are used for comparison, to determine the influence of particle absorption index values on flowfield characteristics. In one simulation, an alternate set of IR absorption index values from measurements of Konopka et al. ${ }^{16}$ are used. These values are generally about one order of magnitude lower than those used in the base simulation. The inclusion of both data sets should allow for evaluation of the sensitivity of simulation results to the large inherent uncertainty in $\mathrm{Al}_{2} \mathrm{O}_{3}$ IR absorption index values. (As discussed by Konopka et al. and by Reed and Calia, ${ }^{2}$ this uncertainty is in part a consequence of an extreme sensitivity to small impurity concentrations, phase composition and lattice defects in solid $\mathrm{Al}_{2} \mathrm{O}_{3}$.) Results are also considered from the two simulations discussed above for which UV absorption index or spectral emissivity values are assumed independent of both temperature and wavelength.

Two additional simulations are also included here for comparison. In the first of these, an anisotropic scattering model $^{6}$ employed in all other cases is disabled, and instead isotropic scattering is assumed. This procedure is equivalent to resetting the average cosine of the scattering angle in the Henyey Greenstein scattering phase function from 0.5 - as taken from Reed et al. ${ }^{17}$ and used in the other simulations - to zero. In the second simulation, the effect of continuum radiation - termed "searchlight emission" - from the interior of the nozzle is neglected, so that all energy bundles used for radiation calculations originate at source particles within the interior of the grid.

In Fig. (12), temperatures for $0.4 \mu \mathrm{m}$ diameter particles along the extraction line are compared among all simulations for which radiation modeling parameters are varied. The corresponding temperature plot for $4 \mu \mathrm{m}$ particles is shown in Fig. (13). Note first that every modification to the radiation model described above has virtually no effect on particle temperatures in the plume nearfield region within a few meters of the nozzle exit. Here the particle energy balance is dominated by the influence of convective heat transfer, and particle residence time in this region is too small for emissive heat loss to have any significant cumulative effect. Any changes to radiation input parameters or modeling approximations should therefore have no impact on particle temperatures in the nearfield plume region, as is observed in Figs. (12) and (13).

In comparing temperatures of both $0.4 \mu \mathrm{m}$ and $4 \mu \mathrm{m}$ particles between the base simulation and those for which elements of flowfield-radiation coupling are disabled, we find that radiative absorption has no significant effect on particle temperatures even in the farfield plume region toward the downstream edge of the simulation domain. In contrast, particle temperatures in this region may increase considerably when the contribution of emissive heat transfer is neglected.

When full radiation-flowfield coupling is included and IR absorption index values are reduced by roughly an order of magnitude to the alternate values of Konopka et al., ${ }^{16}$ the farfield temperatures for both small and large particles are shown in Figs. (12) and (13) to increase significantly. Based on findings of Konopka et al. that the difference between initial and reduced absorption index values represents the typical uncertainty in the IR absorption index range for $\mathrm{Al}_{2} \mathrm{O}_{3}$ particles in a SRM plume flow, we can assume that the difference between temperature curves for the base simulation and the simulation with alternate IR absorption index values corresponds to an unavoidable error margin in the results. 
In contrast, the use of alternate absorption index values in the UV range produces no significant change in particle temperatures, due to the relatively small contribution of UV emission to the total radiative heat transfer rate. Results from the simulations where anisotropic scattering or searchlight emission are neglected also appear nearly identical to those from the base simulation. Both searchlight emission and the angular dependence of scattering probabilities are therefore found to have no significant influence on the temperature of $0.4 \mu \mathrm{m}$ or $4 \mu \mathrm{m}$ diameter particles throughout the plume.

Values of the net radiative energy flux are shown in Fig. (14) for all simulations involving modifications to the radiation model. Note first that no difference is observed between results from the base simulation and those from the simulation where the influence of radiative absorption on flowfield properties is neglected. However, a lack of radiation-flowfield coupling for both absorption and emission is found to significantly increase energy flux values far downstream of the nozzle, as results from the corresponding increase in particle temperatures shown in Figs. (12) and (13).

The choice of values for the IR particle absorption index is found to significantly influence energy flux values through the entire plume. When the lower "alternate" values are used, the net energy flux is considerably reduced in both nearfield and farfield plume regions. This is due to the fact that thermal radiative emission in the relevant temperature range for $\mathrm{Al}_{2} \mathrm{O}_{3}$ particles occurs primarily at IR wavelengths. As spectral emissivity is assumed in the radiation model to scale linearly with the absorption index, any large change to IR absorption index values will result in a comparable change to values of the net radiative energy flux.

We find no significant difference in the net energy flux when UV absorption index values are varied, as a relatively small fraction of emissive heat loss occurs in the UV range. A similar lack of influence on the energy flux is found in the scattering model; results shown in Fig. (14) for the simulation with isotropic scattering are virtually identical to those for the base simulation.

Nozzle searchlight emission is observed in Fig. (14) to account for over $50 \%$ of the net radiative energy flux around the nozzle exit, while providing a negligible contribution to flux values at distances greater than a few meters from the nozzle. Much of the downstream reduction in the radiative energy flux within the plume nearfield region can be attributed to the corresponding decrease in the intensity of radiation emitted inside the nozzle. When nozzle searchlight emission is disabled, a far more gradual downstream reduction in energy flux is found in this region.

Figure (15) shows values of UV spectral radiance at the onboard sensor for all simulations described in this section, and data points from the BSUV-2 flight experiment are plotted for comparison. As mentioned in the discussion of Fig. (6), when either the UV absorption index or emissivity is assumed to be independent of wavelength, significantly better agreement is found with experimental radiance values in the 0.2 to $0.25 \mu \mathrm{m}$ range where a particularly large discrepancy is observed between experimental values and those from the base simulation. The simulation for which both emission and absorption are uncoupled from flowfield calculations shows consistently higher radiance values than most other simulations, as results from the corresponding increase in particle temperatures.

The level of statistical scatter in Fig. (15) is great enough that few definite conclusions can be drawn from any trends observed in the figure. We do find, however, that of all the modifications to the radiation model considered here, only adjustments to the UV absorption index produce a statistically significant effect on calculated UV radiance values. It follows from this comparison that most other modifications to the radiation model cannot be assumed to measurably improve overall simulation accuracy, to the extent that simulation accuracy is established by the level of agreement between experiment and simulation results. Note that no broad conclusions can be drawn here for the general application of the radiation model to high altitude SRM plume flows. If, for example, a much larger scale plume of greater optical thickness had been considered, we expect that modifications to the scattering model would have a far more significant effect on the calculated radiance. The influence of both the scattering model and searchlight emission would also likely be much greater if the sensor field of view was aligned more closely to the nozzle exit plane. ${ }^{17}$

\section{Potential Influence of Soot on Plume Radiation}

In addition to condensed phase particles of $\mathrm{Al}_{2} \mathrm{O}_{3}$, an SRM exhaust plume at high altitude may contain a significant concentration of soot particles. Soot is produced during the combustion process in a wide range of chemical-propellant rocket propulsion systems, and is thought to appear primarily as a result of local chemical nonequilibrium within reacting flow regions. ${ }^{1}$ Due to the complex nature of soot formation, and a general lack of understanding for the relevant physical processes, much uncertainty exists in the characterization of soot as part of the flowfield simulation and radiation analysis of SRM plume flows. However, experimental measurements in a 
high altitude test chamber have shown a relatively high soot content in off-axis regions of a SRM exhaust plume. ${ }^{18}$ Soot has been incorporated into detailed models for the simulation of low altitude SRM plume flows, ${ }^{19}$ but an extensive literature search revealed no efforts at the simulation of high altitude SRM plumes which include consideration of soot radiation.

In both experimental and numerical radiation studies for liquid-propellant rocket plumes, soot is often cited as a major contributor to plume emission characteristics. ${ }^{1,20,21}$ To evaluate the potential contribution of soot to radiation properties for the solid-propellant plume flows of interest, additional simulations are run for the BSUV-2 flow in which soot is included as an additional particle species. In particular, we hope to determine the effect of soot on calculated UV radiance values at the onboard sensor, so that the influence of soot particles on the discrepancy between experimental and numerical results may be quantitatively evaluated.

Based on previous simulations and experimental measurements of soot concentrations in exhaust flows from liquid-propellant rocket engines, ${ }^{1,20}$ we assume a $2 \%$ mass loading of soot at the nozzle exit. Although no corresponding data can be found for an SRM exhaust flow, the $2 \%$ value represents a reasonable - although very rough - estimate of the expected soot concentration in the simulated flowfield. We assume for simplicity that the soot concentration is uniform over the nozzle exit plane, and soot particles are given initial temperatures and velocities which are identical to those used for the gas. While noticeable soot velocity and temperature lags may exist in some regions within the nozzle, the very small mass and heat capacity of soot particles allows these particles to rapidly equilibrate to local gas properties in the plume. The approximation of full equilibration at the nozzle exit should therefore have little or no impact on plume radiation or flowfield properties.

Following the expected soot particle size distribution given by Simmons, ${ }^{1}$ all soot particles are given a diameter of $0.04 \mu \mathrm{m}$. Input parameters for the radiation model include wavelength and temperature-dependent values of both the refractive and absorption indices, based on an analysis of experimental data also compiled by Simmons. ${ }^{1}$

For inclusion of soot in the radiation model, all procedures are nearly identical to those used for $\mathrm{Al}_{2} \mathrm{O}_{3}$ particles. ${ }^{6}$ The only exception is in the calculation of spectral emissivity $\varepsilon_{\lambda}$; the correlation of Reed and Calia ${ }^{2}$ rearranged here as Eq. (1) is not valid for use with soot, so an alternate expression must be found for use in determining soot particle emission and absorption properties. As a starting point, Mie theory gives the following expression ${ }^{1,22}$ for the spectral absorptance $\alpha_{\lambda}$ at a wavelength $\lambda$ for particles of radius $\mathrm{R}_{\mathrm{p}}<\lambda / 2 \pi$ :

$$
\alpha_{\lambda}=\frac{36 \times 10^{-4} \pi \mathrm{nk}}{\left(\mathrm{n}^{2}-\mathrm{k}^{2}+2\right)^{2}+4 \mathrm{n}^{2} \mathrm{k}^{2}} \cdot \frac{f_{\mathrm{v}}}{\lambda}
$$

Here $\mathrm{k}$ is the particle absorption index, $\mathrm{n}$ is the refractive index ( $\mathrm{n}+\mathrm{ki}$ make up the complex index of refraction at the wavelength $\lambda$ ) and $f_{\mathrm{v}}$ is the particle volume fraction, defined as the product of the particle number density $n_{\mathrm{p}}$ and the volume per particle $4 / 3 \pi \mathrm{R}_{\mathrm{p}}{ }^{3}$. (Note that Eq. (2) differs from the formula provided by Simmons ${ }^{1}$ and Plastinin et al. ${ }^{22}$ in the inclusion of the factor $10^{-4}$. This factor is required so that $\alpha_{\lambda} \cdot \lambda$ will be a nondimensional quantity. In both of the references mentioned, $\alpha_{\lambda}$ is given in $\mathrm{cm}^{-1}$ while $\lambda$ has units of $\mu \mathrm{m}$.) The spectral absorptance may also be given as the product of the particle absorption cross section and number density $n_{\mathrm{p}}$.

$$
\alpha_{\lambda}=\pi \mathrm{R}_{\mathrm{p}}^{2} \varepsilon_{\lambda} n_{\mathrm{p}}
$$

By setting the right-hand sides of Eqs. (2) and (3) equal, we can express the spectral emissivity $\varepsilon_{\lambda}$ as a function of the nondimensional ratio $R_{p} / \lambda$.

$$
\varepsilon_{\lambda}=\frac{48 \times 10^{-4} \pi \mathrm{nk}}{\left(\mathrm{n}^{2}-\mathrm{k}^{2}+2\right)^{2}+4 \mathrm{n}^{2} \mathrm{k}^{2}} \cdot \frac{\mathrm{R}_{\mathrm{p}}}{\lambda}
$$

Equation (4) may then be used in radiation modeling procedures to determine emission and absorption characteristics of soot particles.

Two additional simulations are performed to assess the potential influence of soot in the BSUV-2 flow. The first simulation includes soot among the exhaust products as described above, and is in all other ways identical to the base simulation. In the second simulation, $\mathrm{Al}_{2} \mathrm{O}_{3}$ particles are entirely removed, so that the contribution of soot to plume radiation characteristics may be independently evaluated. Note however that the second simulation neglects any effects of soot absorption and scattering on radiation emitted by $\mathrm{Al}_{2} \mathrm{O}_{3}$ particles, so the removal of $\mathrm{Al}_{2} \mathrm{O}_{3}$ particles allows for only an approximate assessment of the influence of soot.

The variation in net radiative energy flux along the extraction line is shown in Fig. (16). Results are presented for the base simulation, the simulation involving both soot and $\mathrm{Al}_{2} \mathrm{O}_{3}$ particles, and the simulation where only soot particles are included. In comparing results between the first two simulations, we find that soot has a very small effect on the net energy flux over the entire length of the extraction line. When soot particles are considered 
independently, the total radiation intensity is found to be about $35 \%$ lower around the nozzle exit, and over two orders of magnitude lower at a distance of about $75 \mathrm{~m}$ downstream.

The divergence in simulation results with downstream distance can be traced in part to the large difference in temperature gradients between soot and $\mathrm{Al}_{2} \mathrm{O}_{3}$ particles, due to convective heat transfer in the nearfield plume region. As mentioned in the discussion of Fig. (3), particle temperature in the plume is generally a strong function of particle size due to the scaling relation between heat capacity and the convective heat transfer rate. Soot particles will therefore cool far more rapidly than the much larger $\mathrm{Al}_{2} \mathrm{O}_{3}$ particles through energy exchange with the surrounding gas. Thermal radiation intensity scales with roughly the fourth power of the source temperature, so the downstream increase in the average temperature difference between soot and $\mathrm{Al}_{2} \mathrm{O}_{3}$ particles corresponds to a far larger difference in radiation intensity contributions from the two types of particles.

Another factor in the downstream increase in differences between simulation results shown in Fig. (16) is the influence of nozzle searchlight emission. As observed in Fig. (14), the relative contribution of searchlight emission to the radiative energy flux decreases continuously with distance from the nozzle exit, so that particle emission and absorption play a comparatively larger role with increasing downstream distance. Searchlight emission has equal intensity for all three simulations considered here, so we expect that differences among these simulations in particle radiation characteristics will result in a downstream increase in the variation between calculated energy flux values.

Figure (17) shows profiles of the plume UV spectral radiance, as measured at the onboard sensor for the base simulation and the two simulations which include soot. By comparing results from these simulations to the experimental values also shown in the figure, we can roughly assess the expected contribution of soot to the discrepancy between experiment and simulation results. An examination of Fig. (17) reveals that, for this particular flow and for the estimated soot concentration used here, soot has no significant influence on the calculated UV radiance values. All differences between radiance values for the base simulation and those for the simulation involving both soot and $\mathrm{Al}_{2} \mathrm{O}_{3}$ particles are within the error bars for statistical scatter, and values for the simulation which includes only soot are uniformly over four orders of magnitude lower.

The fact that soot has such a small effect on plume UV radiance can be attributed to the very small soot mass loading used in the simulations (less than $7 \%$ that of $\mathrm{Al}_{2} \mathrm{O}_{3}$ particles), and particularly to the large difference between soot and $\mathrm{Al}_{2} \mathrm{O}_{3}$ particle temperatures throughout the plume. Note that thermal radiation intensity in the UV range is particularly sensitive to source particle temperatures, while most of the radiative energy flux throughout the simulated flowfield is concentrated at IR wavelengths where thermal radiative emission is a weaker function of temperature. The relative contribution of soot is therefore found to be far smaller in Fig. (17) than in the net radiative energy flux values displayed in Fig. (16).

\section{Summary and Conclusions}

The simulation of high altitude exhaust plumes from solid propellant rockets presents a number of modeling difficulties, and involves consideration of several complex physical processes which are not entirely understood. The work presented here has been performed with the goal of advancing the current state of modeling capabilities for these flows, and assessing the relative influence and interaction between potentially important phenomena. While the simulation scheme described above does not resolve many of the known sources of inaccuracy in the simulation of high altitude SRM plume flows, ${ }^{2}$ it does address several flow physical processes that have received little previous attention in the literature, and allows these processes to be considered together within a unified numerical framework.

In an effort to evaluate the overall accuracy of the proposed scheme, a series of simulations has been performed for a representative SRM plume flow. Comparisons have been made between calculated UV radiance values and measured values from a flight experiment. While no definite conclusions could be drawn from these comparisons, relatively good agreement was observed between simulation and experimental results. Note that a rigorous assessment of simulation accuracy is not possible due a lack of more detailed experimental data, although the level of agreement observed here is an encouraging indication of overall accuracy.

A series of parametric studies involving simulations of the same SRM plume flow have been used to evaluate the influence of several physical processes and input parameters. The first of these studies considered models for gasparticle interaction. Here we found that particle and gas temperatures through much of the plume may be significantly affected by two-way interphase momentum and energy exchange. Values of the particle thermal accommodation coefficient were found to greatly influence flowfield properties as well as radiation intensity in the farfield plume region, while effects of particle rotation were shown to be negligible.

Another study examined models and parameters related to plume radiation. Here we found that only the choice of particle UV absorption index values can account for the discrepancy observed between simulation and 
experimental values of plume spectral radiance in the 0.2 to $0.25 \mu \mathrm{m}$ wavelength range. Most of the disagreement with the experimental data can therefore be traced to a large inherent uncertainty in the determination of absorption index values. Within the IR range, these values were also found to strongly influence particle temperatures and net radiation intensity through much of the plume. Nozzle searchlight emission was shown to significantly contribute to net radiation intensity in the nearfield plume region, while particle radiative emission - but not absorption - was observed to have a considerable effect on particle temperatures further downstream.

A third parametric study considered the potential influence of soot on plume radiation properties. Soot was found to have a very small effect on net radiation intensity throughout the plume, with a contribution relative to that of $\mathrm{Al}_{2} \mathrm{O}_{3}$ particles which decreases continuously with downstream distance. As measured from a simulated onboard sensor, UV spectral radiance values associated with soot are roughly four orders of magnitude smaller than base simulation values, so that the presence of soot may be safely ruled out as a significant contributor to the differences observed between simulation results and experimental data.

\section{Acknowledgments}

The authors gratefully acknowledge the Air Force Research Laboratory at Edwards Air Force Base for financial support of this work, with Tom Smith as technical monitor.

\section{References}

${ }^{1}$ Simmons, F. S., Rocket Exhaust Plume Phenomenology, Aerospace Press, El Segundo, CA, 2000.

${ }^{2}$ Reed, R. A., and Calia, V. S., "Review of Aluminum Oxide Rocket Exhaust Particles," AIAA Paper 93-2819, 1993.

${ }^{3}$ Burt, J. M., and Boyd, I. D., "Development of a Two-Way Coupled Model for Two Phase Rarefied Flows," AIAA Paper 2004-1351, 2004.

${ }^{4}$ Burt, J. M., and Boyd, I. D., "Particle Rotation Effects in Rarefied Two Phase Plume Flows," 24th International Symposium on Rarefied Gas Dynamics, Monopoli, Italy, 2004.

${ }^{5}$ Burt, J. M., and Boyd, I. D., "Monte Carlo Simulation of a Rarefied Multiphase Plume Flow," AIAA Paper 2005-0964, 2005.

${ }^{6}$ Burt, J. M., and Boyd, I. D., “A Monte Carlo Radiation Model for Simulating Rarefied Multiphase Plume Flows,” AIAA Paper 2005-4691, 2005.

${ }^{7}$ Erdman, P. W., Zipf, E., Espy, P., Howlett, C., Levin, D. A., and Candler, G. V., "In-Situ Measurements of UV and VUV Radiation from a Rocket Plume and Re-entry Bow Shock,” AIAA Paper 92-0124, 1992.

${ }^{8}$ Candler, G. V., Levin, D. A., Brandenburg, J., Collins, R., Erdman, P., Zipf, E., Howlett, C., "Comparison of Theory with Plume Radiance Measurements from the Bow Shock Ultraviolet 2 Rocket Flight," AIAA Paper 920125, 1992.

${ }^{9}$ Bird, G. A., Molecular Gas Dynamics and the Direct Simulation of Gas Flows, Clarendon Press, Oxford, 1994.

${ }^{10}$ Breuer, K. S., Piekos, E. S., and Gonzales, D. A., "DSMC Simulations of Continuum Flows,” AIAA Paper 952088, 1995.

${ }^{11}$ Burt, J. M., and Boyd, I. D., "Evaluation of a Particle Method for the Ellipsoidal Statistical Bhatnagar-GrossKrook Equation,” AIAA Paper 2006-0989, 2006.

${ }^{12}$ Boyd, I. D., Chen, G., and Candler, G. V., "Predicting Failure of the Continuum Fluid Equations in Transitional Hypersonic Flows,” Physics of Fluids, Vol. 7, No. 1, 1995, pp. 210-219.

${ }^{13}$ Anfimov, N. A., Karabadjak, G. F., Khmelinin, B. A., Plastinin, Y. A., and Rodinov, A. V., "Analysis of Mechanisms and Nature of Radiation from Aluminum Oxide in Different Phase States in Solid Rocket Exhaust Plumes,” AIAA Paper 93-2818, 1993.

${ }^{14}$ Gallis, M. A., Torczynski, J. R., and Rader, D. J., "An approach for Simulating the Transport of Spherical Particles in a Rarefied Gas Flow via the Direct Simulation Monte Carlo Method," Physics of Fluids, Vol. 13, No. 11, 2001, pp. 3482-3492.

${ }^{15}$ Siegel, R., and Howell, J. R., Thermal Radiation Heat Transfer, Hemisphere Publishing, Washington, 1981.

${ }^{16}$ Konopka, W. L., Reed, R. A., and Calia, V. S., "Measurements of Infrared Optical Properties of $\mathrm{Al}_{2} \mathrm{O}_{3} \mathrm{Rocket}$ Particles," AIAA Paper 83-1568, 1983.

${ }^{17}$ Reed, R. A., Beale, K. S., Neese, D. W., Sherrell, F. G., Roberds, D. W., and Oliver, S. M., "The Effect of Seachlight Emission on Radiation from Solid Rocket Plumes,” AIAA Paper 92-2918, 1992. 
${ }^{18}$ Girata, P. T., and McGregor, W. K., "Particle Sampling of Solid Rocket Motor (SRM) Exhausts in High Altitude Test Cells,” AIAA Paper 83-245, 1983.

${ }^{19}$ Plastinin, Y. A., Karabadzhak, G., and Khmelinin, B., "Ultraviolet, Visible and Infrared Spectra Modeling for Solid and Liquid-Fuel Rocket Exhausts,” AIAA Paper 2001-660, 2001.

${ }^{20}$ Wright, M. J., Rao, R. M., Candler, G. V., Hong, J. S., Schilling, T. A., and Levin, D. A., "Modeling Issues in the Computation of Plume Radiation Signatures,” AIAA Paper 98-3622, 1998.

${ }^{21}$ Garrison, M., Ozawa, T., and Levin, D. A., "An Improved $\mathrm{CO}_{2}, \mathrm{H}_{2} \mathrm{O}$ and Soot Infrared Radiation Models for High Temperature Flows,” AIAA Paper 2005-4777, 2005.

${ }^{22}$ Plastinin, Y., Karabadzhak, G., Khmelinin, B., Baula, G., and Rodionov, A., "Advanced Model for Soot Radiation in the Plume,” AIAA Paper 2002-798, 2002.

Table 1. Particle properties at the nozzle exit.

\begin{tabular}{|l|l|l|l|l|}
\hline $\begin{array}{l}\text { Diameter, } \\
\mu \mathrm{m}\end{array}$ & $\begin{array}{l}\text { Mass flux, } \\
\mathrm{kg} / \mathrm{m}^{2} \mathrm{~s}\end{array}$ & $\begin{array}{l}\text { Temperature, } \\
\mathrm{K}\end{array}$ & $\begin{array}{l}\text { Speed, } \\
\mathrm{m} / \mathrm{s}\end{array}$ & $\begin{array}{l}\text { Liquid mass } \\
\text { fraction }\end{array}$ \\
\hline 0.3 & 0.0443 & 1562 & 2992 & 0.579 \\
\hline 0.4 & 0.0367 & 1634 & 3051 & 0.661 \\
\hline 0.6 & 0.133 & 1834 & 3023 & 0.89 \\
\hline 1 & 0.592 & 2293 & 2973 & 1 \\
\hline 2 & 2.29 & 1920 & 2855 & 0.989 \\
\hline 4 & 7.53 & 2178 & 2674 & 1 \\
\hline 6 & 1.84 & 2407 & 2472 & 1 \\
\hline
\end{tabular}

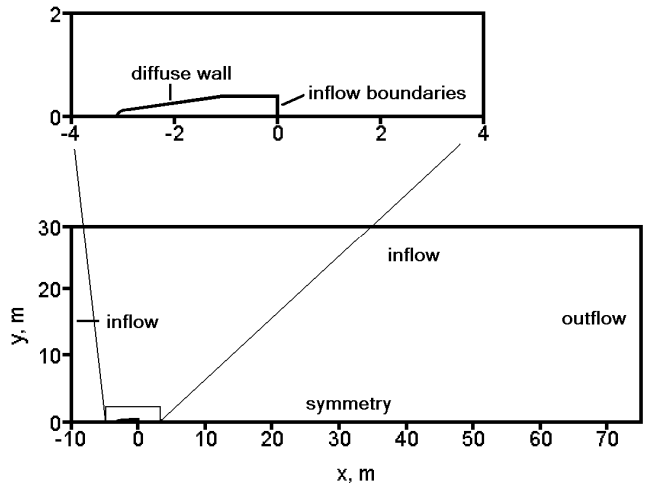

Fig. 1 Grid geometry for simulations of the BSUV-2 flow.

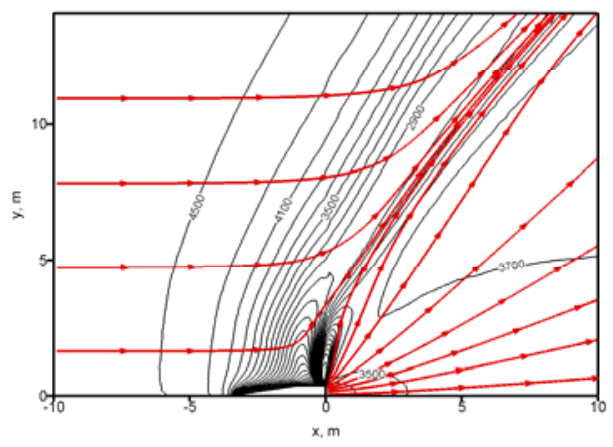

Fig. 2 Gas streamlines and contours of bulk velocity magnitude. Contour line values are given in $\mathbf{m} / \mathbf{s}$.

14

American Institute of Aeronautics and Astronautics 


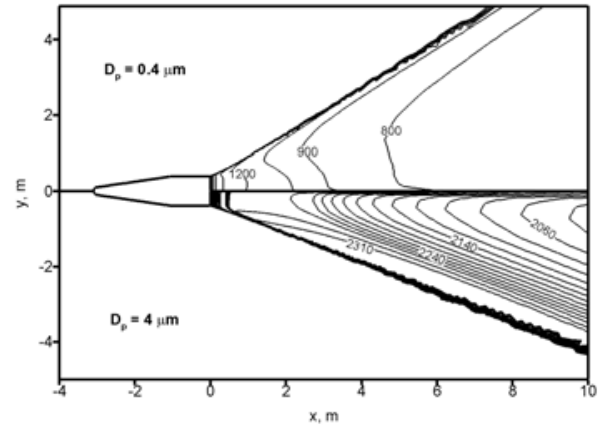

Fig. 3 Close-up view of particle temperature contours. Values are given in $\mathrm{K}$.

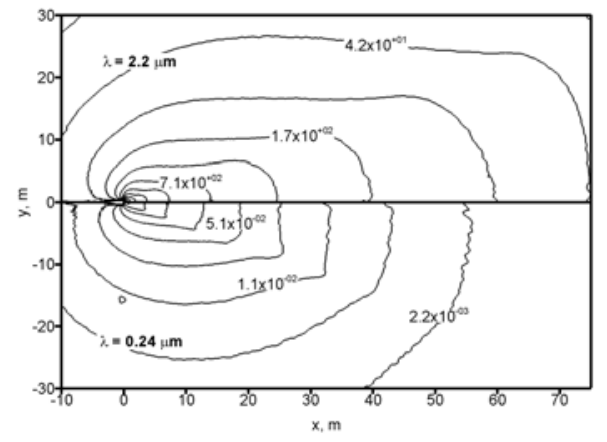

Fig. 5 Contours of the direction-averaged spectral energy flux at wavelengths of $2.2 \mu \mathrm{m}$ and $0.24 \mu \mathrm{m}$. Values are in $\mathrm{W} / \mathrm{m}^{2} \mu \mathrm{m}$.

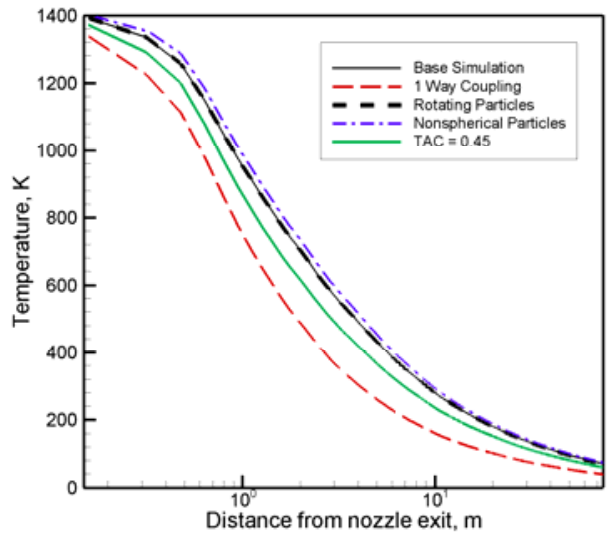

Fig. 7 Gas translational temperature along the extraction line.

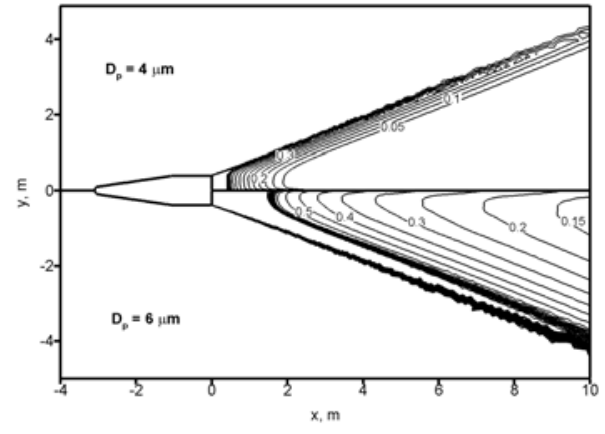

Fig. 4 Contours of liquid mass fraction for 4 and $6 \mu \mathrm{m}$ diameter particles.

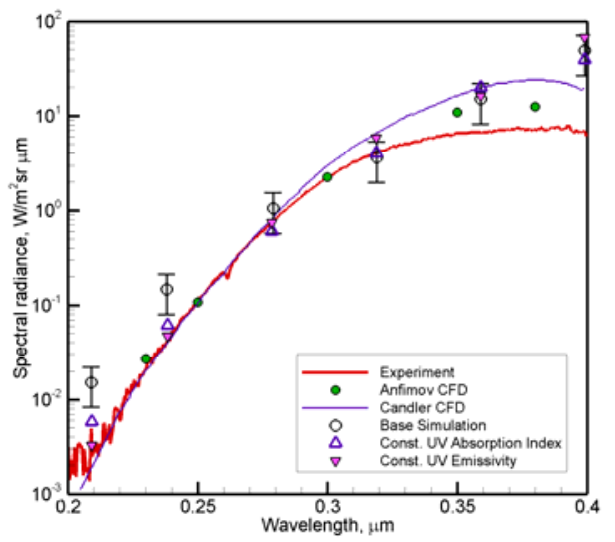

Fig. 6 UV spectral radiance measured at the onboard sensor.

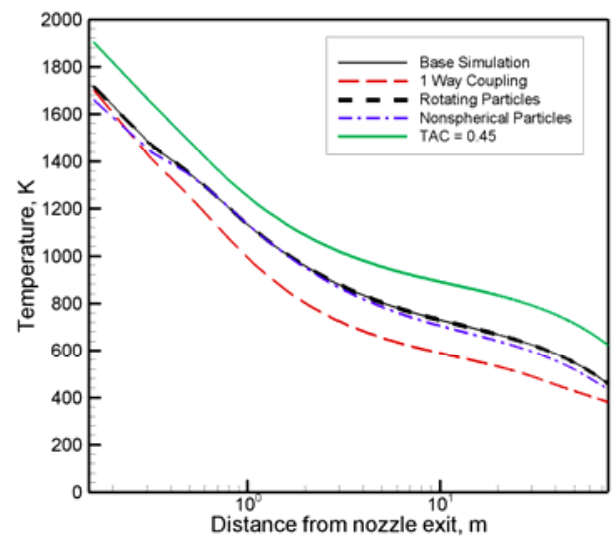

Fig. 8 Temperature variation along the extraction line for $0.4 \mu \mathrm{m}$ particles. 


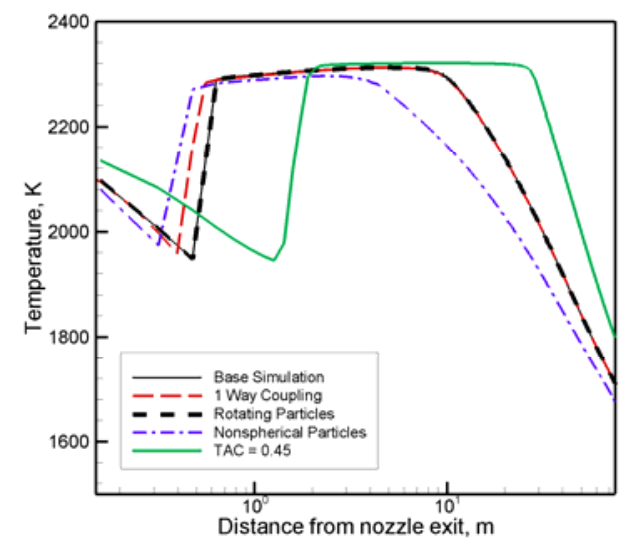

Fig. 9 Temperature variation along the extraction line for $4 \mu \mathrm{m}$ particles.

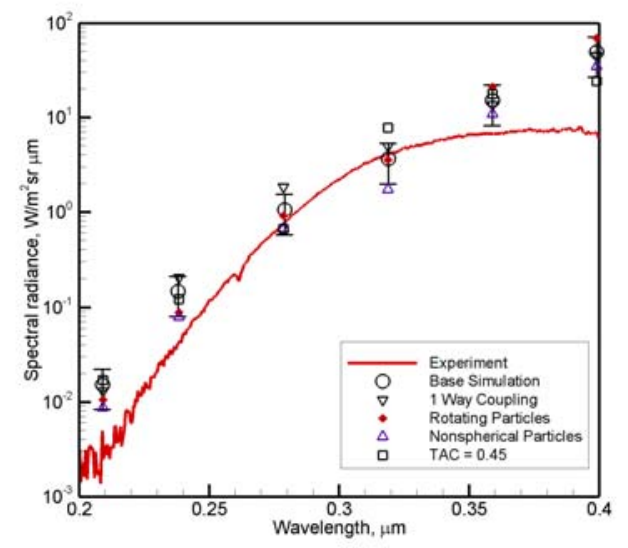

Fig. 11 UV spectral radiance at the onboard sensor.

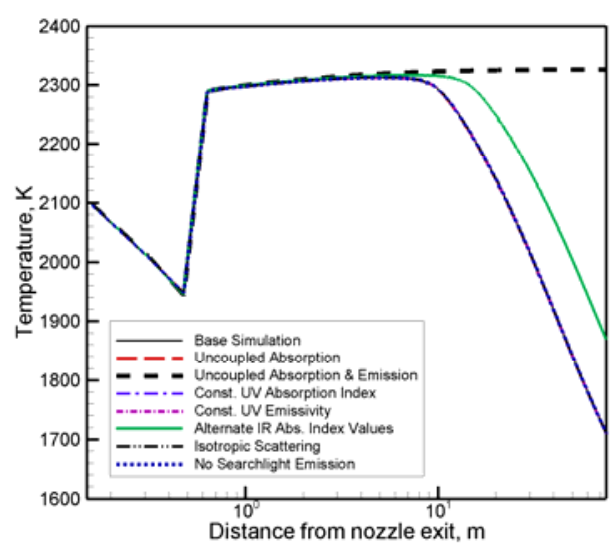

Fig. 13 Temperature of $4 \mu \mathrm{m}$ particles along the extraction line.

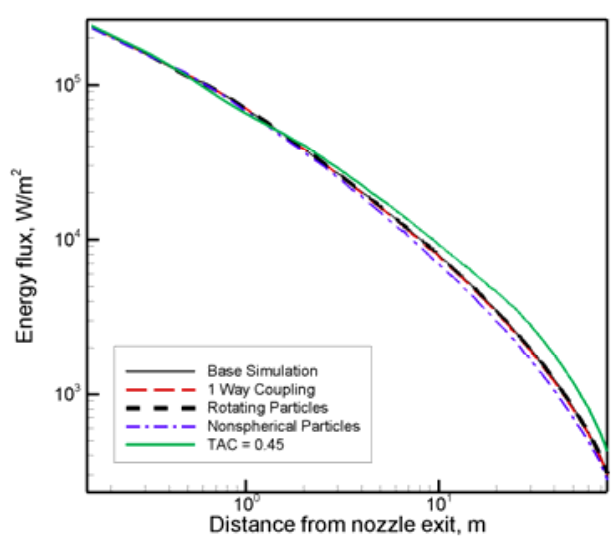

Fig. 10 Net radiative energy flux along the extraction line.

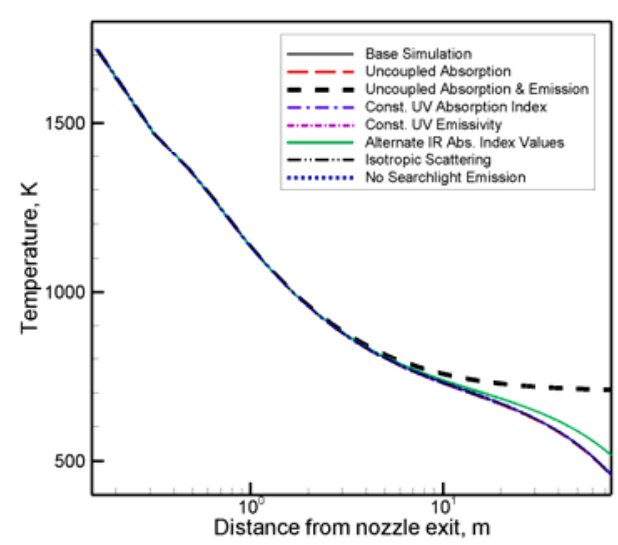

Fig. 12 Temperature of $0.4 \mu \mathrm{m}$ particles along the extraction line.

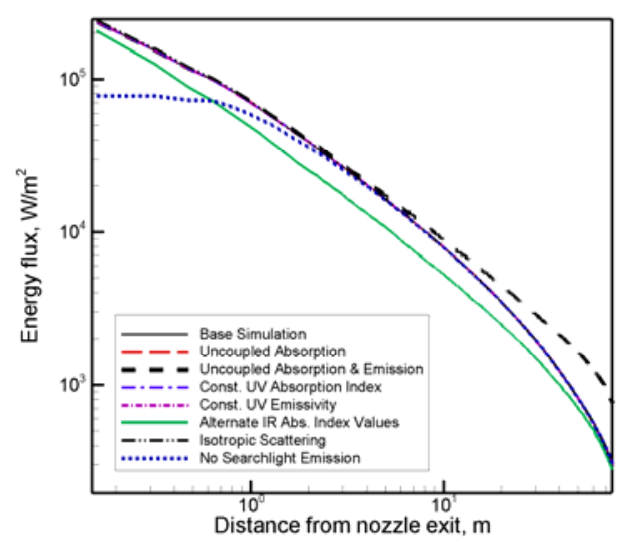

Fig. 14 Variation along the extraction line in the net direction-averaged radiative energy flux. 


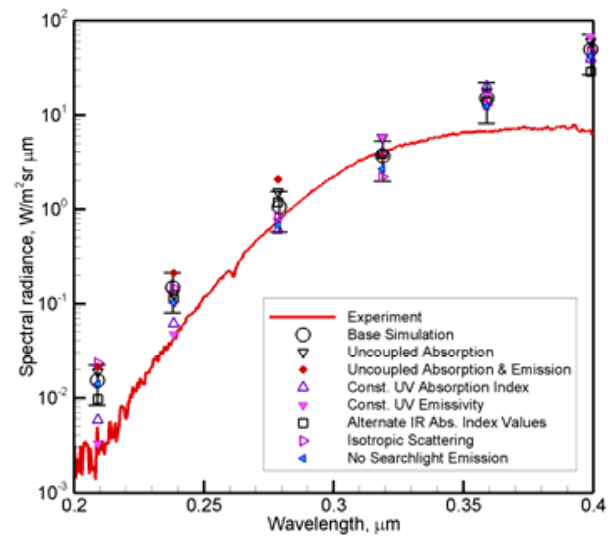

Fig. 15 Dependence of UV spectral radiance on radiation model parameters.

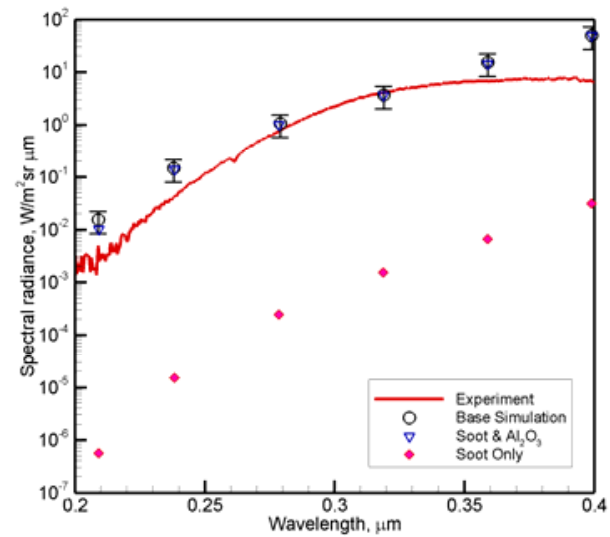

Fig. 17 Effect of soot on UV radiance at the onboard sensor.

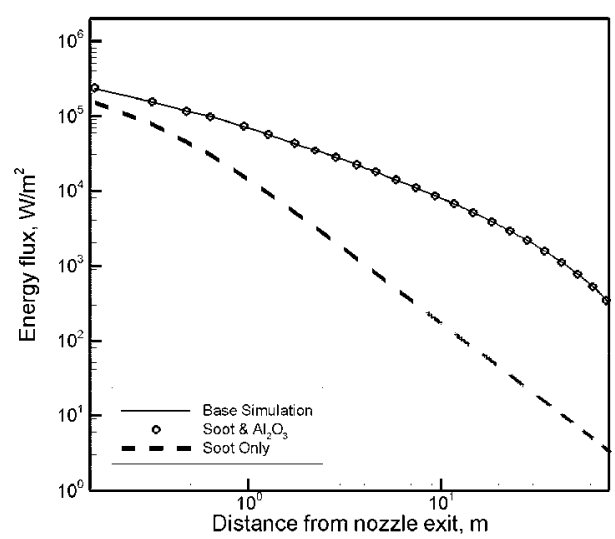

Fig. 16 Effect of soot on the net radiative energy flux along the extraction line. 